\title{
A New Method to Optimize the Maintenance Strategy of HVDC Transmission System
}

\author{
Yuguang Lai, Lin Guan \\ School of Electrical Power, South China University of Technology, Guangzhou 510640, China \\ Email: 281981371@qq.com, 451966005@qq.com
}

Received April, 2013

\begin{abstract}
A method based on fuzzy comprehensive evaluation to optimize the maintenance strategy of HVDC transmission system is proposed to provide quantitative reference for the maintenance arrangements. Firstly, a system based on fuzzy comprehensive evaluation, is established to fuzzy the index results of power system adequacy evaluation, so as to analysis the degree of risk in the operation of power system and the effect brought by the maintenance of HVDC. Then, the proposal on the maintenance of HVDC can be formed through the quantization and evaluation summary of the fuzzy results. The equivalent network of the China Southern Power Grid in 2011, which respectively operates in the circumstances of peak load, general load and low load, demonstrates the HVDC transmission systems in different operation modes have corresponding Overhaul timing. The result shows that the method proposed is valid and in accordance with engineering practice.
\end{abstract}

Keywords: Fuzzy Comprehensive Evaluation; Maintenance Strategy; HVDC

\section{Introduction}

The maintenance of HVDC basically uses the regular maintenance mode which has been introduced from Soviet Union in 1950s. In order to pursue higher energy availability, the operation departments in power grid tend to allow HVDC transmission system to operate continuously, even work with defects, which is not conducive to improve the equipment health.

At present, maintenance strategy researches for power grid are mainly based on the theory of condition-based maintenance. Some paper [1-2] has proposed the condition-based maintenance based on the life-cycle cost, aiming to extend the life cycle of the equipment so as to manage and maintain the grid equipment. Reference [3] introduced the grey hierarchy evaluation method, in which the actual state of the transformer is assessed through the associated hierarchy analysis. What's more, Reference [4] proposed a maintenance decision-making method based on grey correlation degree and TOPSIS. However, the methods above all rely on the improvement of detection, evaluation and diagnosis technique for the equipment status. Currently, condition-based maintenance is still in its infancy and is difficult to be applied in HVDC transmission system.

This paper builds the fuzzy evaluation system to analysis the operational risk of the power grid, and quantita- tively point out the impact on power reliability brought by HVDC outage. Furthermore, it is available to complete a comprehensive evaluation of HVDC maintenance time according to the fuzzy result of power system and risk increased by HVDC maintenance.

\section{Fuzzy Comprehensive Evaluation}

Base on the researches $[5,6]$, it seen to be true that the key failures to give expression to the impact brought by HVDC maintenance are the failures of AC lines that parallel to HVDC. In order to study the reliability of the power grid influenced by HVDC, this paper gets the basic statistics for Power system reliability assessment by enumeration methods.

As for the need to analysis distinctively the failure with different order, this paper proposed a method to assess the risk in grid based on Fuzzy comprehensive evaluation. In fuzzy comprehensive evaluation, the target will be described by several factors, which are called evaluation index set $U$. The evaluation also need comments, which are called comment, set $V$. By using the fuzzy mapping, we can change the evaluation index into membership grades which correspond to comment set. All membership grades of evaluation index can constitute a fuzzy matrix $R$, in which the fuzzy relation $U \times V$ could be presented. What's more, the fuzzy result $B$ 
can be achieved.

\section{The Assessment about the Operational Risk and Impact of HVDC Maintenance Based on Fuzzy Comprehensive Evaluation}

In order to deal with different failure, this paper will build a double level fuzzy model (almost the same model for two targets). The operational risk and impact of HVDC maintenance are the first level evaluation objects, and the evaluation indexes in first model will become the evaluation objects of second model.

\subsection{Evaluation Index}

In this paper, the evaluation index of first level model are LOLP, total lost load and the overload frequency, and the evaluation index of second level model are the LOLP and overload frequency in different order failure including $\mathrm{N}-1, \mathrm{~N}-2, \mathrm{~N}-3$ and $\mathrm{N}-4$ fault set. The statistics of evaluation index can be gained by following methods.

\subsection{The Index Weight and the Comment of Evaluation Set}

As for the fuzzy comprehensive evaluation on the operational risk of grid and the impact from the HVDC transmission system outage, the comment of evaluation set and its quantitative score is shown in Table 1:

The index weight reflects the contribution of evaluation indicator to the evaluation object. It can gain the judgment matrix according to the expertise, and then the index weight $\omega_{1}=(0.3,0.5,0.2)$ in first level of the two evaluation model could be determined basing on the AHP (Analytic hierarchy process). Again, the index weight in second level is $\omega_{2}=(0.4,0.3,0.2,0.1)$. The above weight corresponds to the previous evaluation indexes in turn.

\subsection{Evaluation Fuzzy}

The process of the calculation of the fuzzy matrix is also a process of evaluation fuzzification, including numerical standardization of evaluation index and fuzzy mapping.

Table 1. The comment sets of fuzzy evaluation.

\begin{tabular}{cccccc}
\hline $\begin{array}{c}\text { The comment } \\
\text { level }\end{array}$ & $v_{1}$ & $v_{2}$ & $v_{3}$ & $v_{4}$ & $v_{5}$ \\
\hline $\begin{array}{c}\text { Operational } \\
\text { risk of grid }\end{array}$ & Low & $\begin{array}{c}\text { Relatively } \\
\text { low }\end{array}$ & Mid & $\begin{array}{c}\text { Relatively } \\
\text { high }\end{array}$ & High \\
& 1 & 2 & 3 & 4 & 5 \\
$\begin{array}{c}\text { Impact from } \\
\text { HVDC out- } \\
\text { age }\end{array}$ & Small & $\begin{array}{c}\text { Relatively } \\
\text { small }\end{array}$ & Mid & $\begin{array}{c}\text { Relatively } \\
\text { great }\end{array}$ & Great \\
\hline
\end{tabular}

1) Numerical standardization

The evaluation index is divided into effective and cost index, and all the indexes discussed by this paper are the cost index. The smaller result of the index, the higher reliability of power gird and the lower fuzzy risk it is. The method to standardize index is,

$$
t=\left(t^{\prime}-\min t^{\prime}\right) /\left(\max t^{\prime}-\min t^{\prime}\right)
$$

where, $t$ is the result after standardization, $t^{\prime}$ is the index before standardization, which refers to LOLP, total lost load and overload frequency.

2) Fuzzy mapping

After numerical standardization, it is substituted in subordinate function of fuzzy mathematics. Then the result can be obtained. This paper selects isosceles triangle subordinate function and $\Gamma$ subordinate function, and combines them flexibly as follow,

$$
\begin{gathered}
r\left(v_{1}\right)= \begin{cases}1-\frac{t^{\prime}}{0.25}, & 0 \leq t^{\prime}<0.25 \\
0, & \text { other }\end{cases} \\
r\left(v_{5}\right)= \begin{cases}1-e^{-\left(\frac{t^{\prime}-0.75}{0.25}\right)}, & 0.75 \leq t^{\prime} \\
0, & t^{\prime}<0.75\end{cases} \\
r\left(v_{4}\right)= \begin{cases}0, & t^{\prime}<0.5 \\
\frac{t^{\prime}}{0.25}-2, & 0.5 \leq t^{\prime}<0.75 \\
e^{-\left(\frac{t^{\prime}-0.75}{0.25}\right)}, & 0.75 \leq t^{\prime}\end{cases} \\
r\left(v_{k}\right)= \begin{cases}\frac{t^{\prime}-0.25(\mathrm{k}-2)}{0.25}, & 0.25(k-2) \leq t^{\prime}<0.25(k-1) \\
\frac{0.25 \mathrm{k}-t^{\prime}}{0.25}, & 0.25(k-1) \leq t^{\prime}<0.25 k \\
0, & \text { other }\end{cases}
\end{gathered}
$$

where, $k=2$, 3. $r\left(v_{i}\right)$ is the result of fuzzy mapping which corresponds to the evaluation comment, and $i=$ $1,2, \ldots, 5$. We can calculate the fuzzy evaluation matrix according to the fuzzy model with double level .What is important to emphasize is that the outcome of evaluation incurrent operation mode may exceed the historical maximum with the growth of the load. Therefore, the maximum of variable in subordinate function is greater than 1 , so as to guarantee that the fuzzy mapping is compatible with such situations.

\subsection{Fuzzy Compositional Operations}

Combine with the fuzzy weight vector and fuzzy evaluation matrix, the fuzzy result of the evaluation objectives 
can be completed, which can be expressed as,

$$
\begin{gathered}
b_{i}=\frac{\sum_{j}^{3} a_{j} r_{j i}}{\sum_{i}^{5} \sum_{j}^{3} a_{j} r_{j i}}, \quad i=1,2, . ., 5 \\
\sum_{j}^{n} a_{j}=1
\end{gathered}
$$

where $b_{i}$ shows the relative-membership grade of evaluation $B$ corresponding to comment $i$.

\section{The Judgment for Maintenance Time of HVDC}

The judgment for maintenance time of HVDC can be achieved basing on the overhaul criterion, which is help to optimize the actual maintenance strategy. According to quantization of each comment in Table 1, we can get the quantitative result by,

$$
\begin{gathered}
C=\sum_{i=1}^{5} b_{i} v_{i} \\
\text { or } \quad C=\sum_{i=1}^{5} b_{i} v_{i} \times 100 \%
\end{gathered}
$$

Furthermore, it is suggested taking comment in which the quantitative score is near to result $C$ as the risk comment. When the fuzzy result (risk comment or quantitative score) meet one of the following conditions, the decision may be recommended for the HVDC maintenance. The conditions are,

1) The risk comment for the operation of power grid is "low", and the impact brought by the maintenance of HVDC meets the requirement that $C \leq 40 \%$;

2) The risk comment for the operation of power grid is "relatively low", and the impact brought by the maintenance of HVDC meets the requirement that $C \leq 30 \%$;

The risk comment for the operation of power grid is "mid", and the impact brought by the maintenance of HVDC meets the requirement that $C \leq 20 \%$;

\section{Case and Analysis}

\subsection{Base case}

To verify the reasonability and practicability of the evaluation system, this article selects the China Southern Power Grid 500kV equivalent network in 2011 as an example to analysis. Equivalent network respectively have peak, general and low load level operation mode. And the three kinds of operation mode's parameter are shown in the following Table 2.

The equivalent gird under three operational modes approximately represents the typical operation of CSG in 2011. The HVDC including Tian-Guang, Gao-Zhao,
Table 2. The presentation of example.

\begin{tabular}{cccc}
\hline Operational mode & Peak load & General load & Low load \\
\hline Gen & 51 & 51 & 49 \\
500kV AC line & 357 & 357 & 351 \\
220kV AC line & 25 & 25 & 22 \\
Total PG (MW) & 125026.3 & 106272.4 & 81587.4 \\
Total PD (MW) & 81836.8 & 69561.28 & 55557.4 \\
Total PQ (MVar) & 40097.4 & 30674.51 & 21770.1 \\
\hline
\end{tabular}

Xing-An and Chu-Sui, which are in control of CSG, all are in bipolar running state.

\subsection{Case Result}

The proposed method was tested on China Southern Power Grid, which was under a high level operation mode in 2011.Under the operation mode, the fuzzy evaluation matrix of the grid operational risk can be calculated using the equation (2)-(5)as followed:

$$
R_{r 1}=\left[\begin{array}{l}
0.0000 .0000 .0000 .5720 .428 \\
0.0000 .0000 .0000 .5500 .450 \\
0.0000 .0000 .1670 .5560 .277
\end{array}\right]
$$

The evaluation matrix shows that the LOLP and the total lost load index completely belong to the "Relatively high" and "High" level of risk, and the overload frequency also reflects a high risk result. Fuzzy comprehensive evaluation result set can be calculated using the equation (6) as followed:

$$
B_{r 1}=(0.000,0.000,0.033,0.558,0.409)
$$

Similarly, the fuzzy comprehensive evaluation result under the medium and small level operational mode can also be obtained.

As for reliability assessment about the impacts of HVDC transmission system outage on grid, this paper selected Tian-Guang and Xing-An HVDC as research objects. Take the case of Tian-Guang HVDC unipolar outage. When the grid system was in a high level operation mode, the fuzzy evaluation matrix of Tian-Guang HVDC unipolar outage is:

$$
R_{e 1}=\left[\begin{array}{l}
0.1140 .4410 .2640 .1810 .000 \\
0.0350 .9650 .0000 .0000 .000 \\
0.4120 .4210 .1670 .0000 .000
\end{array}\right]
$$

Similarly, we can also obtain:

$$
B_{e 1}=(0.134,0.699,0.113,0.054,0.000)
$$

The risk impact of quantization results can be obtained using the formula (8). In the high level operation mode, the effects of the Tian-Guang and Xing-An HVDC with single and bipolar outage can be summarized as shown in Table 3:

Analyzing the risk impact of HVDC system outage in 
the way of other operation mode is similar to the proposed method as above. According to overhauling timing criterion, we obtain the following Tables 4-6.

Results from Tables 4-6 show that, when the power grid was in the way of large operation mode, it was in a higher level of risk originally, and the grid is in need of the HVDC system as power support, unfitting to carry out regular maintenance.

Results from Tables 4-6 also show that, when the power grid was in the way of large operation mode, it was in a higher level of risk originally, and the grid is in need of the HVDC system as power support, unfitting to carry out regular maintenance.

When the power grid was in the way of medium operation mode, it was in a lower level of risk. The risk effect of Tian-Guang HVDC unipolar and bipolar outage and Xing-An HVDC unipolar outage is less than 30\%, so there is lower risk impact on the power grid to carry out regular maintenance initiatively. However, Xing-An HVDC bipolar outage still has higher impact, so it does not recommend to outage.

When the power grid was in the way of lower operation mode, the operational risk is in the ideal minimum level. All of the HVDC system single and double pole outage has a very small influence on the power grid. The impact of Tian-Guang HVDC the single and double polar outage and Xing-An HVDC unipolar outage is equivalent to no impact at all, that is to say, it meets heat stability under all the fault condition. Consequently, the HVDC system is fully equipped for planned outage.

Table 3. The results of the impact from HVDC outage.

\begin{tabular}{ccc}
\hline HVDC operation & Fuzzy result $B_{e}$ & $\begin{array}{c}\text { Quantization } \\
\text { result } C_{e}\end{array}$ \\
\hline T-G unipolar outage & $(0.134,0.699,0.112,0.054,0.000)$ & $27.18 \%$ \\
T-G bipolar outage & $(0.059,0.174,0.672,0.074,0.021)$ & $45.56 \%$ \\
X-A unipolar outage & $(0.068,0.410,0.431,0.069,0.021)$ & $39.13 \%$ \\
X-A bipolar outage & $(0.023,0.040,0.111,0.144,0.682)$ & $85.52 \%$ \\
\hline
\end{tabular}

Table 4. The proposal on the maintenance of HVDC in the mode of peak load.

\begin{tabular}{|c|c|c|c|c|}
\hline HVDC Operation & Operational risk & Risk effect & Suggestion & Reason \\
\hline T-G unipolar outage & & $27.18 \%$ & No maintenance & \\
\hline T-G bipolar outage & & $45.56 \%$ & No maintenance & \\
\hline & High & & & Operational risk all are high \\
\hline $\mathrm{X}$-A unipolar outage & & $39.13 \%$ & No maintenance & \\
\hline X-A bipolar outage & & $85.52 \%$ & No maintenance & \\
\hline
\end{tabular}

Table 5. The proposal on the maintenance of HVDC in the mode of general load.

\begin{tabular}{|c|c|c|c|c|}
\hline HVDC Operation & Operational risk & Risk effect & Suggestion & Reason \\
\hline T-G unipolar outage & & $8.95 \%$ & maintenance & Meet condition 2 \\
\hline \multirow[t]{2}{*}{ T-G bipolar outage } & & $18.48 \%$ & maintenance & Meet condition 2 \\
\hline & Relatively low & & & \\
\hline X-A unipolar outage & & $12.34 \%$ & maintenance & Meet condition 2 \\
\hline X-A bipolar outage & & $42.97 \%$ & No maintenance & Risk effect is high \\
\hline
\end{tabular}

Table 6. The proposal on the maintenance of HVDC in the mode of low load.

\begin{tabular}{|c|c|c|c|c|}
\hline HVDC Operation & Operational risk & Risk effect & Suggestion & Reason \\
\hline T-G unipolar outage & & $0.00 \%$ & maintenance & Meet condition 1 \\
\hline T-G bipolar outage & & $0.00 \%$ & maintenance & Meet condition 1 \\
\hline \multicolumn{5}{|c|}{ Low } \\
\hline X-A unipolar outage & & $0.00 \%$ & maintenance & Meet condition 1 \\
\hline X-A bipolar outage & & $3.75 \%$ & maintenance & Meet condition 1 \\
\hline
\end{tabular}




\section{Conclusions}

In this paper, fuzzy comprehensive evaluation results are basically consistent with the practical engineering, and its calculation method too simple and intuitive to meet the need of application engineering. The maintenance recommendations can be obtained from the evaluation result and maintenance time criterion, so the theoretical results can be used to optimize the maintenance strategy of HVDC.

\section{REFERENCES}

[1] M. Nima, T. Kato and Y. Suzuoki, "Life-cycle-cost Evaluation of Degradation Diagnosis for Cables,” Electrical Insulating Materials, 2005, pp. 737-740.

[2] Z. N. Ma, L. H. Zhong, K. Lu and C. J. Xia, "Study on the Maintenance Strategy of Power Equipment Based on the Optimal Life Cycle Cost," Power System Protection and Control, 2011, pp. 34-39.

[3] H. Xiong, C. X. Sun, Y. Zhang, et al., “A Hierarchical Grey Evaluation Model for Operation Condition of Power Transformers," Automation of Electric Power Systems, 2007, pp. 55-60.

[4] L. J. Yang, X. F. Xiong and Y. Zhang, "Research on Condition-based Maintenance Policy of Electric Power Equipment Based on Grey Correlation Degree and TOPSIS,” Power System Protection and Control, 2009, pp. 74-78.

[5] G. Q. Wan, Z. Ren, S. F. Wang, et al., "Reliability Evaluation Algorithm of Power System Based on Outage Margin of Safety State Space Pruning," Automation of Electric Power Systems, 2007, pp. 22-26.

[6] J. S. Zhang, Y. Zhang and Z. G. Wu, "Analysis on Regional Load Margin of Guangdong,” Power System Technology, 2006, pp. 30-34. 